\title{
Cross-Coupling of [2-Aryl-1,1,2,2-tetrafluoroethyl](trimethyl)silanes with Aryl Halides
}

\author{
Miriam O’Duill, Emmanuelle Dubost, Lukas Pfeifer, and Véronique Gouverneur* \\ Chemistry Research Laboratory, University of Oxford, 12 Mansfield Road, OX1 3TA Oxford, U.K. \\ Supporting Information Placeholder

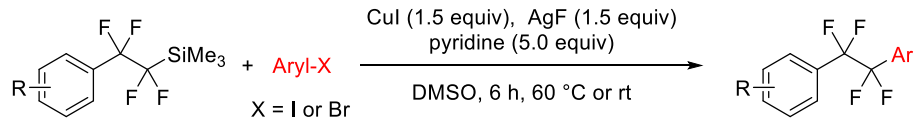 \\ (22 examples)
}

\begin{abstract}
The synthesis of arylCF $\mathrm{CF}_{2} \mathrm{SiMe}_{3}$ and their reactivity in cross-coupling reactions with aryl iodides and aryl bromides to afford a range of 1,1,2,2-tetrafluoro-1,2-arylethanes is reported. The use of pyridine as an alternative to phenanthroline, and the ability to carry out the reaction at $60^{\circ} \mathrm{C}$ or room temperature are the key features of this $\mathrm{Cu}-\mathrm{Ag}$ mediated cross-coupling methodology. The chemistry is compatible with (hetero)aryl halides, offering a platform to develop products of interest in material and medicinal chemistry.
\end{abstract}

The Ruppert-Prakash reagent $\left(\mathrm{CF}_{3} \mathrm{TMS}\right)$ is a stable and easy to handle commercially available reagent widely employed for late stage trifluoromethylation. ${ }^{1}$ Metal-mediated crosscoupling strategies with this reagent have been extensively studied, ${ }^{2}$ more recently with a focus on copper-mediated processes with aryl halides. ${ }^{3-4}$ The use of this class of reagents to install extended perfluoroalkyl chains is limited to (pentafluoroethyl)trimethylsilane $\left(\mathrm{C}_{2} \mathrm{~F}_{5} \mathrm{TMS}\right)$ and some selected studies employing more functionalized perfluorinated trimethylsilane derivatives (Scheme 1a). ${ }^{4 a, g-i}$ We noted a single

Scheme 1. Synthesis of 1,1,2,2-Tetrafluoro-1,2,arylethanes. 1) Ruppert-Prakash reagents for cross-coupling with aryl halides

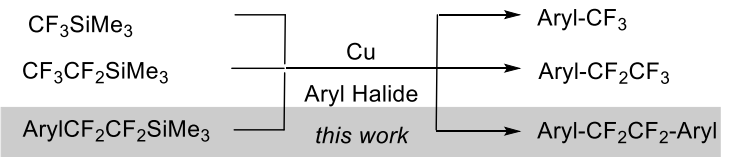

2) Copper mediated synthetic approaches towards $\mathrm{ArCF}_{2} \mathrm{CF}_{2} \mathrm{Ar}$

$$
\text { Aryl- } \mathrm{CF}_{2} \mathrm{CF}_{2} \mathrm{Br} \stackrel{\mathrm{Cu} \text { (excess), DMSO }}{\stackrel{\text { Aryl iodide }}{130-140{ }^{\circ} \mathrm{C}, 24 \mathrm{~h}}} \text { Aryl- } \mathrm{CF}_{2} \mathrm{CF}_{2} \text {-Aryl }
$$

example of a copper-mediated cross-coupling reaction of [2aryl-1,1,2,2-tetrafluoroethyl](trimethyl)silane (arylCF $\mathrm{CF}_{2}-$ TMS) with 1-iodo-4-nitrobenzene, a reaction affording 1(1,1,2,2-tetrafluoro-2-(4-nitrophenyl)ethyl)- $1 \mathrm{H}$-pyrazole in $25 \%$ yield. ${ }^{5}$ The product formed in this reaction belongs to a class of highly valuable 1,1,2,2-tetrafluoro-1,2-arylethane derivatives presenting with a $\mathrm{CF}_{2} \mathrm{CF}_{2}$ unit flanked by two aryl (or heteroaryl) groups; however the low yield for this isolated reaction implies narrow applicability. The usefulness of these compounds to access novel perfluorinated materials such as liquid-crystalline compounds ${ }^{6}$ has encouraged the development of a range of alternative methods for their synthesis, using precursors other than [2-aryl-1,1,2,2-tetrafluoroethyl](trimethyl)silanes. Strategies featuring late stage fluorination are known but suffer from harsh reaction conditions. ${ }^{7}$ More recently, 2-bromo-1,1,2,2-tetrafluoroethylarenes were found to be suitable for cross-coupling reactions with aryl iodides in the presence of an excess of copper, but these couplings require temperatures higher than $130{ }^{\circ} \mathrm{C}$ and extended reaction times (Scheme 1b, eq 2). ${ }^{8}$ Ogoshi et al. disclosed an elegant alternative strategy based on the generation of 2-aryl-1,1,2,2tetrafluoroethylcopper complexes from $[\mathrm{CuO} t \mathrm{Bu}]_{4}$, tetrafluoroethylene (TFE) and arylboronic esters (Scheme 1b, eq 3). These complexes were successfully used in cross-coupling reactions with aryl iodides; the use of gaseous TFE is not ideal for common research laboratory settings, and the sensitivity of the $[\mathrm{CuO} t \mathrm{Bu}]_{4}$ precursor may be limiting as a glove box is preferable for handling. ${ }^{9}$ Our research program on $\mathrm{Cu}$-mediated ${ }^{18} \mathrm{~F}$-radiochemistry for Positron Emission Tomography (PET) applications is currently expanding with the development of new methodologies for the labeling of perfluorinated arenes. ${ }^{10}$ This program led us to prepare [2-aryl-1,1,2,2-tetrafluoroethyl](trimethyl)silanes and develop an efficient protocol for the synthesis of 1,1,2,2-tetrafluoro-1,2-arylethanes via copper/silver-mediated cross-coupling with a range of aryl halides (Scheme 1). Herein, we disclose this operationally simple and mild reaction and exemplify its scope on a range of (hetero)aryl iodides and bromides.

This study began with the synthesis of the model [2-aryl1,1,2,2-tetrafluoroethyl](trimethyl)silane 1a, which was prepared from the parent aryl bromide following a three-step procedure (Scheme 2). First, the Schlosser Grignard reagent derived from 4-bromo-1,1'-biphenyl was reacted with methyl chlorodifluoroacetate at $-40{ }^{\circ} \mathrm{C}$ in THF. Treatment of the resulting ketone with DAST at $60{ }^{\circ} \mathrm{C}$ afforded 4-(2-chloro1,1,2,2-tetrafluoroethyl)-1,1'-biphenyl in $65 \%$ overall yield 
Scheme 2. Synthesis and X-ray structure of [2-(Biphenyl-4yl)-1,1,2,2-tetrafluoroethyl](trimethyl)silane 1a.
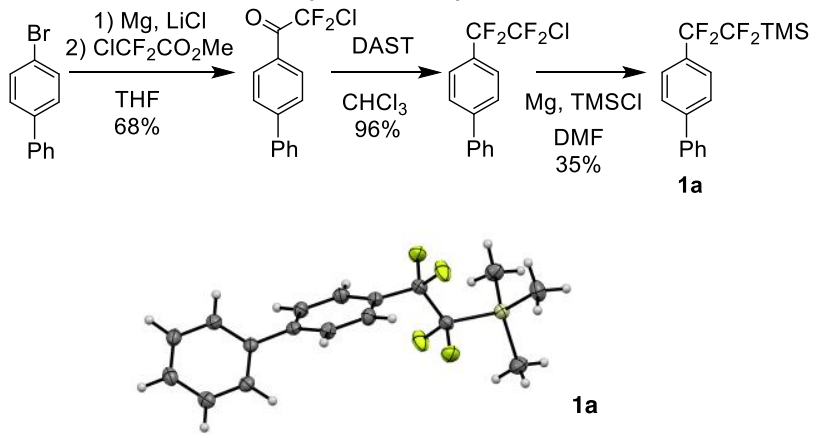

after two steps. The subsequent reaction, a magnesium-mediated trimethylsilylation, was less efficient but this process was readily scalable, delivering more than two grams of [2-(biphenyl-4-yl)-1,1,2,2-tetrafluoroethyl](trimethyl)silane $\mathbf{1 a}$; this compound is a white crystalline solid found suitable for single crystal X-ray diffraction analysis. ${ }^{11,12}$ The additional [2aryl-1,1,2,2-tetrafluoroethyl](trimethyl)silanes $\mathbf{1 b}$ and $\mathbf{1 c}$ used in this study were prepared following a similar reaction sequence. For 1c, lithium halogen exchange was preferable to Grignard formation for the trimethylsilylation step. ${ }^{12}$

In the first instance, the reactivity of $\mathbf{1 a}$ was probed with a benchmark reaction, a fluoride-mediated addition to enolizable and non-enolizable aldehydes (Scheme 3).

Scheme 3. Reactivity of [2-(Biphenyl-4-yl)-1,1,2,2-tetrafluoroethyl](trimethyl)silane 1a with Aldehydes. ${ }^{a}$
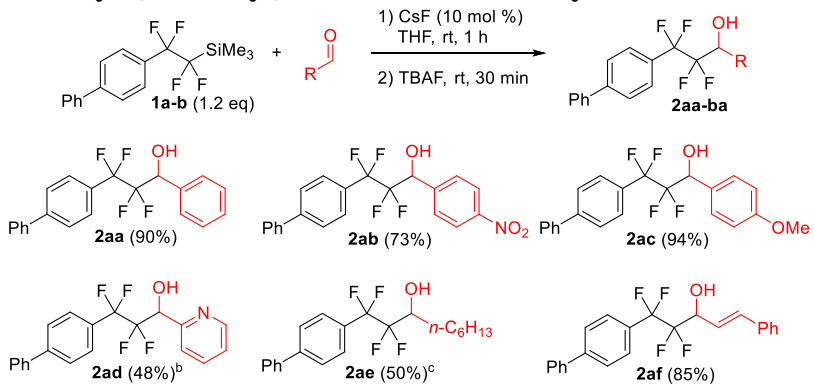

${ }^{a} 1.2$ equiv of $1 \mathrm{a}$ and 1.0 equiv of aldehyde; yields of isolated product. ${ }^{b} 4-(1,1,2,2$-Tetrafluoroethyl)-1,1'-biphenyl was formed as side-product (40\%). ${ }^{c}$ 4-(1,1,2,2-Tetrafluoroethyl)-1,1'-biphenyl (30\%) and 4-(1,2,2,2-tetrafluoroethyl)-1,1'-biphenyl (20\%) were formed as side-products.

The addition of $\mathbf{1 a}$ (1.2 equiv) to benzaldehyde (1.0 equiv) was accomplished at room temperature in THF in the presence of $10 \mathrm{~mol} \% \mathrm{CsF}$. The resulting silylated alcohol was subjected to deprotection using TBAF. The desired compound was isolated in $90 \%$ yield. Electron poor and electron rich benzaldehydes are tolerated but the reaction proved less efficient with pyridine 2-carboxaldehyde and hexanal, affording 2ad and 2ae in $48 \%$ and $50 \%$ yields respectively. For reactions giving the desired products in yields inferior to $70 \%$, protodesilylation of 1a leading to 4-(1,1,2,2-tetrafluoroethyl)1,1 '-biphenyl was observed as a competitive side reaction and an additional product identified as 4-(1,2,2,2-tetrafluoroethyl)-1,1'-biphenyl was formed when using hexanal. ${ }^{13}$ Only traces of 4-(1,2,2-trifluorovinyl)-1,1'-biphenyl resulting from elimination were detectable in the crude reaction mixtures.
We focused next on the Cu-mediated cross-coupling of 1a with 1-iodo-2-methoxy-4-nitrobenzene (Table 1).

Table 1. Optimization studies for the Cross-Coupling of 1a with 1-Iodo-2-methoxy-4-nitrobenzene. ${ }^{a}$

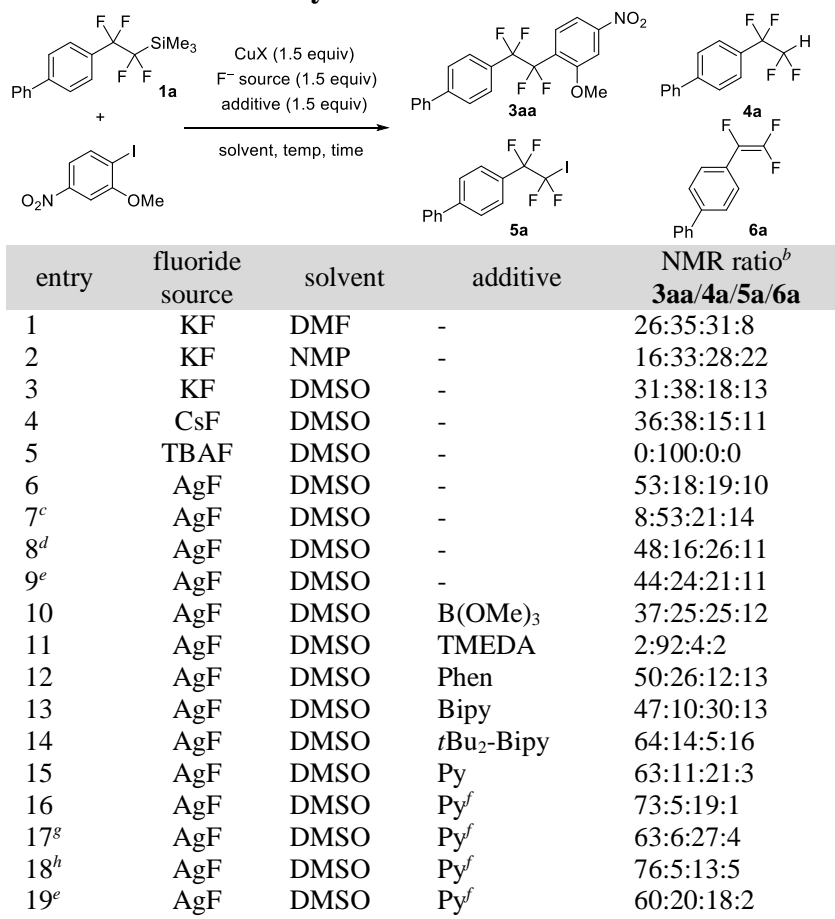

a Standard conditions: 1.0 equiv 1-iodo-2-methoxy-4-nitrobenzene, 1.2 equiv 1a, 1.5 equiv fluoride source, 1.5 equiv of $\mathrm{CuI}$, 1.5 equiv additive (if applicable), $0.25 \mathrm{M}$ in solvent, $60{ }^{\circ} \mathrm{C}, 16 \mathrm{~h}$. TMEDA $=N, N, N^{\prime}, N^{\prime}$-tetramethyl-1,2-ethylenediamine; Phen = 1,10-phenanthroline; Bipy = 2,2'-bipyridine; $t \mathrm{Bu}_{2}$-Bipy $=4,4$ '-ditert-butyl-2,2'-bipyridine; Py $=$ pyridine. ${ }^{b}$ Determined by ${ }^{19} \mathrm{~F}$ NMR by integration of the product peak(s) using $\mathrm{PhCF}_{3}$ as the internal standard. ${ }^{c}$ Reaction with $\mathrm{CuCl} .{ }^{d}$ Reaction with $\mathrm{CuBr} .{ }^{e}$ $20 \mathrm{~mol} \%$ of CuI. ${ }^{f} 5.0$ equiv of pyridine. ${ }^{g} \mathrm{rt}$ for $6 \mathrm{~h} .{ }^{h} 6 \mathrm{~h}$ reaction time.

Our investigation began with the coupling of $\mathbf{1 a}$ and our model aryl iodide in DMF with 1.5 equiv of $\mathrm{KF}$ and $\mathrm{CuI}$ at 60 ${ }^{\circ} \mathrm{C}$ for $16 \mathrm{~h}$ (Table1, entry 1$)$. These conditions led to the desired product 3aa in $26 \%$ yield along with $35 \%$ of $4-(1,1,2,2-$ tetrafluoroethyl)-1,1'-biphenyl $\mathbf{4 a}$ resulting from competitive protodesilylation. The two additional side products observed in the crude reaction mixture were the iodo derivative 5a formed in $31 \%$ yield along with $18 \%$ of alkene 6a. A similar product distribution was obtained using NMP, but the use of DMSO proved beneficial (Table 1, entries 2-3). AgF was the most efficient activator affording the desired coupling product in 53\% yield (Table 1, entry 6). The cooperative effect of silver in the $\mathrm{Cu}$-catalyzed trifluoromethylation of aryl iodides with $\mathrm{CF}_{3} \mathrm{TMS}$ has been reported for other systems by Weng and co-workers. ${ }^{4 \mathrm{e}}$ Alternative sources of $\mathrm{Cu}(\mathrm{I})$ such as $\mathrm{CuBr}$ or $\mathrm{CuCl}$ were less effective (Table 1 , entries 7-8). ${ }^{14}$ The reaction did proceed with a catalytic amount of $\mathrm{CuI}$, however a substantial amount of by-product formation was observed (entry 9). Several additives were considered next. With the Ruppert-Prakash reagent $\mathrm{CF}_{3} \mathrm{SiMe}_{3}, \mathrm{~B}(\mathrm{OMe})_{3}$ was shown to stabilize the $\mathrm{CF}_{3}$ anion in copper mediated cross-coupling, thus minimizing the formation of protodesilylated by-product; ${ }^{4 j}$ no beneficial effect was observed with $1 \mathbf{a}$ (Table 1, entry 10). As 
anticipated, we found that 1,10-phenanthroline and bipyridine were superior to TMEDA, but these ligands afforded product 3aa in only low to moderate conversion (Table 1, entries 1113); the more electron rich 4,4'-di-tert-butyl-2,2'-bipyridine ligand gave 3aa in 64\% (Table 1, entry 14) and pyridine afforded 3aa in $63 \%$ (Table 1, entry 15). Cost-effective pyridine was identified as the best additive for cross-coupling (Table 1, entries 15-16). The use of pyridine as a preferential ligand for copper-mediated cross-coupling methodologies for perfluoroalkylation is not common, but its advantage over other ligands has been documented in the context of flow chemistry. ${ }^{15}$ Monitoring the reaction by NMR indicated that the starting material was consumed after $6 \mathrm{~h}$ (Table 1, entry 17). Applying our best conditions consisting of $\mathrm{CuI}$ (1.5 equiv), $\mathrm{AgF}$ ( 1.5 equiv), pyridine (5.0 equiv) in DMSO at $60{ }^{\circ} \mathrm{C}$ for $6 \mathrm{~h}$, 3aa was isolated in $78 \%$ yield (Table 1, entry 18). Similar conditions using $20 \mathrm{~mol} \%$ of $\mathrm{CuI}$ instead of 1.5 equiv led to inferior results, so these conditions using sub-stoichiometric amount of $\mathrm{CuI}$ were not retained to study the scope of this cross-coupling reaction (Table 1, entry 19).

The substrate scope was investigated next (Scheme 4). Numerous functionalized aryl iodides underwent cross-coupling with 1a. Ketone, nitro, cyano, ether, ester, and bromo substituents are well tolerated with good conversions obtained for both electron-donating and electron-withdrawing substitu-

Scheme 4. Copper-mediated Cross Coupling of 1a with (Hetero)aryl Iodides ${ }^{\text {a }}$

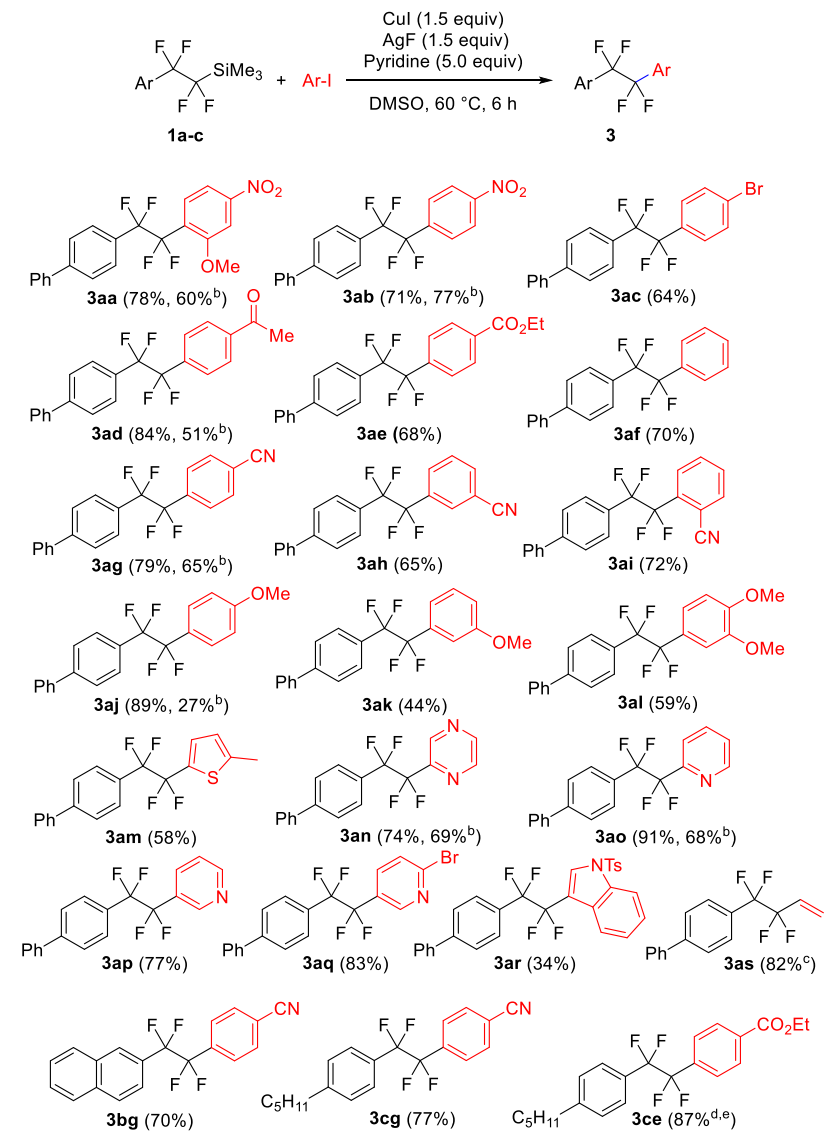

a 1.2 equiv of 1 and 1.0 equiv of aryl iodide ( $0.2 \mathrm{mmol} \mathrm{scale})$; All yields are for isolated products. ${ }^{b}$ Reaction performed at room temperature for $6 \mathrm{~h}^{c}{ }^{c}$ chemical purity $91 \% .{ }^{d} 0.5 \mathrm{mmol}$ scale. ${ }^{c}$ chemical purity $90 \%$. ents. This methodology can be extended to a vinyl iodide as well as a range of heteroaryl iodides including thiophene, pyrazine, indole and pyridine derivatives. The reaction also proceeds with alternative 2-substituted trimethyl(1,1,2,2-tetrafluoroethyl)silanes, as exemplified by the synthesis of $\mathbf{3 b g}, \mathbf{3 c g}$ and 3ce. Compound 3ce is an advanced precursor for the synthesis of a liquid crystalline compound. ${ }^{6 \mathrm{c}} \mathrm{We}$ noted that this protocol allowed for the coupling of $\mathrm{CF}_{3} \mathrm{CF}_{2} \mathrm{TMS}$ with 1iodo-2-methoxy-4-nitrobenzene and $\mathrm{HCF}_{2} \mathrm{TMS}$ with 1-tertbutyl-4-iodobenzene affording the desired products in $82 \%$ (yield of isolated product) and $59 \%\left({ }^{19} \mathrm{~F}\right.$ NMR yield), respectively. This is an improvement over current methods reported in the literature because of the mildness of our reaction conditions. ${ }^{4 \mathrm{~h}, 16}$ The cross-coupling of $\mathbf{1 a}$ with aryl iodides could also be performed at room temperature, but the yields of the isolated products were generally lower under these conditions (Scheme 4). The diaryl derivatives 3ac, 3aq and 3as stand out as candidates for further derivatization via cross-coupling or metathesis.

The difference in availability and price of (hetero)aryl iodides and bromides prompted us to study the coupling of representative (hetero)aryl bromides (Scheme 5). These reactions

\section{Scheme 5. Copper-mediated Cross Coupling of 1a with} (Hetero)aryl Bromides. ${ }^{a}$
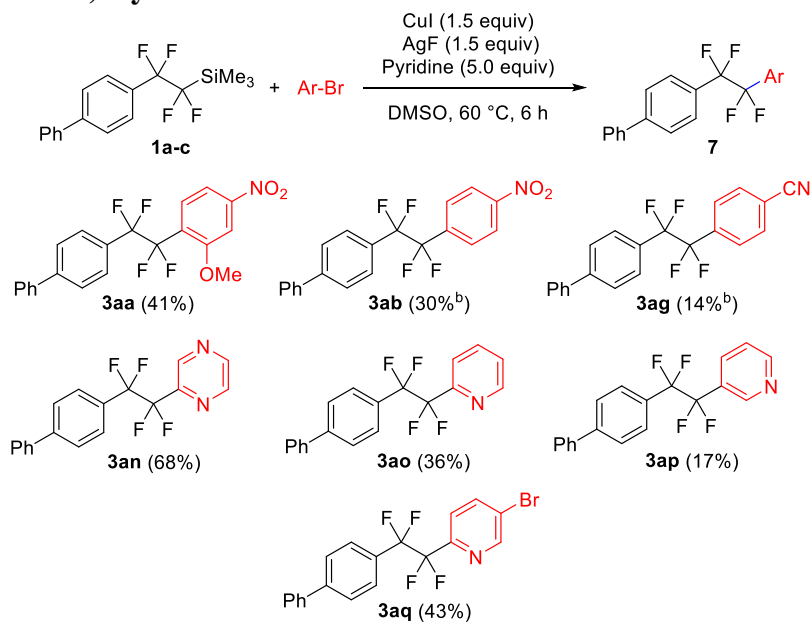

${ }^{a} 1.2$ equiv of $\mathbf{1 a}$ and 1.0 equiv of aryl iodide $(0.2 \mathrm{mmol}$ scale); yields of isolated products unless stated otherwise. ${ }^{b}$ ${ }^{19} \mathrm{~F}$ NMR yields, determined by integration of the product peak(s) using $\mathrm{PhCF}_{3}$ as the internal standard.

were performed using $\mathrm{CuI}$ ( 1.5 equiv), $\mathrm{AgF}$ ( 1.5 equiv), pyridine (5.0 equiv) in DMSO at $60{ }^{\circ} \mathrm{C}$ for $6 \mathrm{~h}$. We found that this reaction does not proceed for electron rich cross-coupling partners such as 1-bromo-4-methoxybenzene. For electron deficient aryl bromides, cross-coupling proceeded under the reaction conditions applied to aryl iodides with yields of isolated products reaching up to $68 \%$. 2-Bromopyridine was more reactive than 3-bromopyridine, a reactivity order allowing for the exclusive formation of product 3aq from 2,3-dibromopyridine.

In summary, we have developed a simple synthetic procedure for the generation of 1,1,2,2-tetrafluoro-1,2-arylethanes from the reaction of stable arylCF $\mathrm{CF}_{2} \mathrm{SiMe}_{3}$ Ruppert-Prakash type reagents with (hetero)aryl iodides or bromides. These reactions are an improvement over current fluoroalkylation reactions due to the mildness of the reaction conditions applied. 
However, improved routes towards arylCF $\mathrm{CF}_{2} \mathrm{SiMe}_{3}$ will be necessary to progress this methodology from research to process. The use of pyridine as an alternative to phenanthroline and the ability to carry out the reaction at $60{ }^{\circ} \mathrm{C}$ or room temperature for aryl iodides are the key features of this cross-coupling methodology. An additional characteristic is the range of (hetero)aryl halides amenable to cross-coupling under such mild reaction conditions. We anticipate that this process will facilitate research programs focusing on the discovery of high performance materials.

\section{ASSOCIATED CONTENT}

\section{Supporting Information}

Experimental procedures and full spectroscopic data for all new compounds. This material is available free of charge via the Internet at http://pubs.acs.org

\section{AUTHOR INFORMATION}

\section{Corresponding Author}

*E-mail: veronique.gouverneur@chem.ox.a.uk.

\section{Notes}

The authors declare no competing financial interest.

\section{ACKNOWLEDGMENT}

Financial support was provided by the BBSRC (studentship to M. O'D.), the ARC (SAE20131200603, E.D.) and the European Union (H2020-MSCA-IF-2014-658405 for a Fellowship to E.D and FP7-PEOPLE-2012-ITN-316882 for a studentship to L. P.). The authors thank Dr. Amber L. Thompson, University of Oxford, for her assistance with the single crystal X-Ray diffraction analysis of $1 \mathbf{a}$.

\section{REFERENCES}

(1) (a) Prakash, G. K. S.; Yudin, A. K. Chem. Rev. 1997, 97, 757. (b) Liu, X.; Xu, C.; Wang, M.; Liu, Q. Chem. Rev. 2015, 115, 683 and references therein.

(2) (a) Tomashenko, O. A.; Grushin, V. V. Chem. Rev. 2011, 111, 4475 and references therein. (b) Wu, X.-F.; Neumann, H.; Beller, M. Chem. Asian J. 2012, 7, 1744 and references therein.

(3) Liu, T.; Shen, Q. Eur. J. Org. Chem. 2012, 6679.

(4) (a) Urata, H.; Fuchikami, T. Tetrahedron Lett. 1991, 32, 91. (b) Dubinina, G. G.; Furutachi, H.; Vicic, D. A. J. Am. Chem. Soc. 2008, 130, 8600. (c) Dubinina, G. G.; Ogikubo, J.; Vicic, D. A. Organometallics 2008, 970, 6233. (d) Oishi, M.; Kondo, H.; Amii, H. Chem. Commun. 2009, 1909. (e) Weng, Z.; Lee, R.; Jia, W.; Yuan, Y.; Wang, W.; Feng, X.; Huang, K. Organometallics 2011, 30, 3229. (f) Tomashenko, O. A.; Escudero-Adán, E. C.; Martínez Belmonte, M.; Grushin, V. V. Angew. Chemie 2011, 123, 7797. (g) Morimoto, H.; Tsubogo, T.; Litvinas, N. D.; Hartwig, J. F. Angew. Chem. Int. Ed. Engl. 2011, 50, 3793. (h) Hafner, A.; Bräse, S. Adv. Synth. Catal. 2013, 355, 996. (i) Mormino, M. G.; Fier, P. S.; Hartwig, J. F. Org. Lett. 2014, 16, 1744. (j) Gonda, Z.; Kovács, S.; Wéber, C.; Gáti, T.; Mészáros, A.; Kotschy, A.; Novák, Z. Org. Lett. 2014, 16, 4268.

(5) Petko, K. I.; Kot, S. Y.; Yagupolskii, L. M. J. Fluor. Chem. 2008, 129, 301.

(6) (a) Kirsch, P.; Bremer, M.; Huber, F.; Lannert, H.; Ruhl, A.; Lieb, M.; Wallmichrath, T. J. Am. Chem. Soc. 2001, 123, 5414. (b) Kirsch, P.; Huber, F.; Lenges, M.; Taugerbeck, A. J. Fluorine Chem. 2001, 112, 69. (c) Kirsch, P.; Huber, F.; Krause, J.; Heckmeier, M.; Pauluth, D. U.S. Patent 20030230737 A1, December 2003. (d) Kirsch, P.; Bremer, M. ChemPhysChem 2010, 11, 357.
(7) (a) Zupan, M.; Pollak, A. J. Org. Chem. 1974, 39, 2646. (b) York, C.; Prakash, G. K. S.; Olah, G. a. J. Org. Chem. 1994, 59, 6493. (c) Singh, R. P.; Majumder, U.; Shreeve, J. M. J. Org. Chem. 2001, 66, 6263. (d) Chang, Y.; Tewari, A.; Adi, A.-I.; Bae, C. Tetrahedron 2008, 64, 9837. (e) Gatenyo, J.; Rozen, S. J. Fluor. Chem. 2009, 130, 332.

(8) (a) Zhu, J.; Ni, C.; Gao, B.; Hu, J. J. Fluor. Chem. 2015, 171, 139. (b) Watanabe, Y.; Konno, T. J. Fluor. Chem. 2015, 174, 102.

(9) Saijo, H.; Ohashi, M.; Ogoshi, S. J. Am. Chem. Soc. 2014, 136, 15158.

(10) (a) Huiban, M.; Tredwell, M.; Mizuta, S.; Wan, Z.; Zhang, X.; Collier, T. L.; Gouverneur, V.; Passchier, J. Nat. Chem. 2013, 5, 941. (b) Tredwell, M.; Preshlock, S. M.; Taylor, N. J.; Gruber, S.; Huiban, M.; Passchier, J.; Mercier, J.; Génicot, C.; Gouverneur, V. Angew. Chem. Int. Ed. 2014, 53, 7751. (c) Emer, E; Twilton, J.; Tredwell, M.; Calderwood, S.; Collier, L. T.; Liégault, B.; Taillefer, M.; Gouverneur, V. Org. Lett. 2014, 16, 6004.

(11) CCDC 1401715. (a) Cosier, J.; Glazer, A. M. J. Appl. Cryst. 1986, 19, 105. (b) Otwinowski, Z.; Minor, W. Macromolecular Crystallography Part A; Carter, C. W., Jr.; Sweets, R. M. Eds.; Methods in Enzymology, Vol. 276; Academic Press: New York, 1997, p. 307. (c) Palatinus, L.; Chapuis, G. J. Appl. Chrystallogr. 2007, 40, 786. (d) Betteridge, P. W.; Carruthers, J. R.; Cooper, R. I.; Prout, K.; Watkin, D. J. J. Appl. Crystallogr. 2003, 36, 1487. (d) Cooper, R. I.; Thompson, A. L.; Watkin, D. J. J. Appl. Crystallogr. 2010, 43, 1100. (e) Parois, P.; Cooper, R. I.; Thompson, A. L. Chem. Cent. J. 2015, 9:30. (f) Thompson, A. L.; Watkin, D. J. J. Appl. Crystallogr. 2011, 44, 10172.

(12) For details, see the Electronic Supporting Information.

(13) 4-(1,2,2,2-Tetrafluoro-ethyl)-1,1'-biphenyl is likely formed by regioselective addition of fluoride onto 4-(1,2,2-trifluorovinyl)$1,1^{\prime}$-biphenyl, a product resulting from competitive elimination.

(14) The formation of side-product $\mathbf{5 a}$ for these reactions performed in the absence of copper iodide indicates that the substrate can serve as the source of iodine.

(15) Chen, M.; Buchwald, S. L. Angew. Chem. Int. Ed. 2013, 52, 11628.

(16) Fier, P. S.; Hartwig, J. F. J. Am. Chem. Soc. 2012, 134, 5524. 